\title{
Paired-angle-rotation scanning optical coherence tomography forward-imaging probe
}

\author{
Jigang Wu, Michael Conry, Chunhui Gu, Fei Wang, Zahid Yaqoob, and Changhuei Yang \\ Department of Electrical Engineering, California Institute of Technology, Pasadena, California, 91125
}

\begin{abstract}
Received December 22, 2005; accepted January 31, 2006; posted February 14, 2006 (Doc. ID 66880)
We report a novel forward-imaging optical coherence tomography (OCT), needle-probe paired-angle-rotation scanning OCT (PARS-OCT) probe. The probe uses two rotating angled gradient-index lenses to scan the output OCT probe beam over a wide angular arc $\left(\sim 19^{\circ}\right.$ half-angle $)$ of the region forward of the probe. Among other advantages, this probe design is readily amenable to miniaturization and is capable of a variety of scan modes, including volumetric scans. To demonstrate the advantages of the probe design, we have constructed a prototype probe with an outer diameter of $1.65 \mathrm{~mm}$ and employed it to acquire four OCT images, with a $45^{\circ}$ angle between adjacent images, of the gill structure of a Xenopus laevis tadpole. The system sensitivity was measured to be $93 \mathrm{~dB}$ by using the prototype probe with an illumination power of $450 \mu \mathrm{W}$ on the sample. Moreover, the axial and the lateral resolutions of the probe are 9.3 and 10.3-12.5 $\mu \mathrm{m}$, respectively. (C) 2006 Optical Society of America

OCIS codes: $170.4500,170.3880,120.5800,170.2150,110.2350,120.3890$.
\end{abstract}

Over the past decade the development of various endoscopic optical coherence tomography (OCT) probes has greatly extended the application range of this high-resolution biomedical imaging technique. ${ }^{1-5}$ Most probe implementations can be divided into two groups based on their scan modes-side imaging ${ }^{1-3}$ and forward imaging. ${ }^{4,5}$ In side-imaging OCT probes the actual scan actuators can generally be located far from the probe tips, which enables very narrow bore side-imaging OCT probes to be built; the smallest reported side-imaging probe ${ }^{2}$ has an outer diameter of $0.4 \mathrm{~mm}$. We note that all side-imaging OCT probes are essentially $2 \mathrm{D}$ in nature, although the combination of side imaging and the back-and-forth translation of the probe may be used to generate $3 \mathrm{D}$ images. In comparison, forward-imaging probes are generally more complicated in design and require the location of the actuators to be at or very near the probe tips. ${ }^{4,5}$ Unsurprisingly, forward-imaging OCT probes that have been reported are relatively large in probe diameter. The smallest reported forward-imaging probe has a diameter of $2.4 \mathrm{~mm}$ and is capable of rendering $2 \mathrm{D}$ scans. ${ }^{4}$ Therefore the research pursuit of a narrower (diameter of $1.5 \mathrm{~mm}$ or smaller) forwardimaging OCT probe design with a wide field of view is a worthy one. Such a probe can be applied in a wide range of clinical procedures, e.g., anesthesiology procedures and breast core biopsies, ${ }^{6}$ where having a forward depth-resolved image ahead of a surgical needle can greatly aid clinicians in guiding the needle to the right locale.

In this Letter we present a new design for a forward-imaging OCT needle probe-the pairedangle-rotation scanning OCT (PARS-OCT) probe. This probe design utilizes a pair of angle-cut rotating gradient-index (GRIN) lenses to deflect and scan the OCT probe beam across the forward region ahead of the probe tip. In this design the scan actuation system may be located away from the probe tip, much like in the case of the side-imaging OCT probe described in Ref. 1; thus, the miniaturization of the probe is straightforward. In addition, this probe de- sign can achieve a large ratio of the forward-scan arc length to the probe diameter. This parameter is especially relevant for clinical probe considerations, as a clinician will desire as wide a scan range as possible and as small a probe size as possible. We note that the use of a small GRIN lens or a GRIN fiber as a probe has been reported by several other groups. ${ }^{7,8}$ Our probe system is unique in that it permits largeangle directional scanning of the output probe beam while maintaining a large effective emissioncollection numerical aperture. As a point of reference, a well-designed probe based on a pair of GRIN lenses with $112 \mu \mathrm{m}$ diameter with a maximum deflection angle of $20^{\circ}$ can have an initial beam width that is $34 \%$ of the probe diameter.

The operating principle of the PARS-OCT probe is as follows. The PARS-OCT probe channels the input OCT probe light from a single-mode fiber through the first GRIN lens [see Figs. 1(a) and 1(b)]. The light beam exits from the other face of the GRIN lens, which is cut at an angle $\psi$. The beam then enters the second GRIN lens through an identically angle-cut face of the GRIN lens. Finally, the beam exits the second GRIN lens and focuses at a point ahead of the probe. The exact focal point is determined by the pitches of the two GRIN lenses. Amongst other good choices, an appropriate choice will be to have a slightly longer than a 1/4 pitch GRIN lens for the first and a shorter than a 1/4 pitch GRIN lens for the second; this choice results in a weakly focused beam between the two GRIN lenses and a tightly focused exit beam with the desired working distance. For completeness, we shall define the orientations of the two GRIN lenses by angles $\zeta_{1}$ and $\zeta_{2}$, the angles between the projections of vectors $\hat{r}_{1}$ and $\hat{r}_{2}$, respectively, in the image plane and the $x$ axis [see Fig. 1(a)]. We shall define the direction of the output light beam by its polar angle $\theta$ that it makes with the $z$ axis and its azimuthal angle $\varphi$; an angle of $\theta=0 \mathrm{im}$ plies that the exit beam propagates along the $z$ axis.

A fan sweep of the output beam in the $x-z$ plane [shown vertically in Fig. 1(d)] can be performed by 


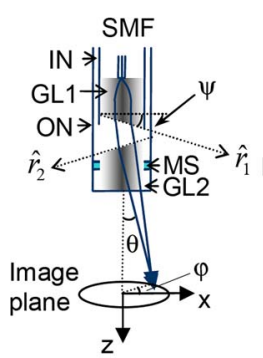

(a)

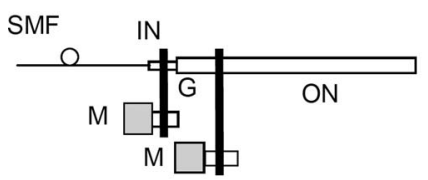

(c)
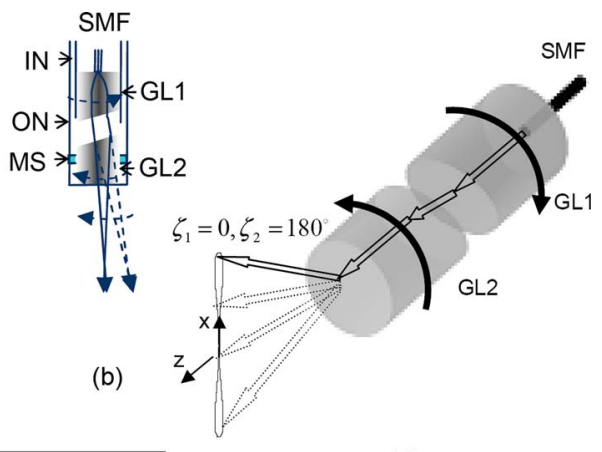

(d)
Fig. 1. (Color online) Schematic of the forward-coneimaging needle probe. (a) The case when there is an angle between the two angled surfaces of the GRIN lenses; the exit laser beam is tilted; (b) The case when the two angled surfaces of the GRIN lenses are parallel; the exit laser beam is undeviated; (c) PARS-OCT probe setup; (d) profile of PARS-OCT B-scan mode. SMF, single-mode fiber; GL1, GRIN lens 1; GL2, GRIN lens 2; IN, inner needle; ON, outer needle; MS, metal sleeve; M, motor; G, gear.

simply rotating the two GRIN lenses in opposite directions at the same angular speed from the starting position where the two GRIN lenses are oriented, such that $\zeta_{1}=0, \zeta_{2}=180^{\circ}$. This scan pattern can be understood by taking a closer look at the initial output beam orientation when $\zeta_{1}=0, \zeta_{2}=180^{\circ}$ [see Fig. 1(a)]. In this case the exit beam from the first GRIN lens is deflected toward the positive $x$-axis direction. Note that the second GRIN lens with $\zeta_{2}=180^{\circ}$ further deflects the beam in the same direction. In Fig. 1(d), this is shown as upward deflection. When we rotate the two GRIN lenses by an equal and opposite amount $\left(\Delta \zeta_{1}=-\Delta \zeta_{2}>0\right)$, the upward deflection of the exit beam from the first GRIN lens will lessen, and the beam will lean to the right if we view the probe head on. The shifting of the beam to the right will be compensated by the second GRIN lens, which conveys an equal but opposite shift to the beam; the upward-deflection contribution of the beam by the second GRIN lens will lessen as well. The net effect is a smaller upward deflection of the beam and little or no horizontal shifting. Continued rotations of the GRIN lenses will eventually result in the GRIN lenses orientation of $\zeta_{1}=\zeta_{2}=90^{\circ}$. In this configuration [see Fig. 1(b)] the two GRIN lenses compensate for each other's deflection of the beam and result in an output beam that is undeflected. Further rotation of the GRIN lenses will then deflect the beam downward. A complete $180^{\circ}$ rotation of the GRIN lens will therefore result in a vertical sweep of the output beam from its up position to its down position-a fan sweep or an effective OCT B scan.

There are three major advantages associated with this probe design. First, by attaching the GRIN lenses to separate concentric needle shafts, we can actuate the GRIN lenses rotations by simply turning the needle shafts. This can be done with actuators that are located far from the probe tip. Second, be- cause of the novel scanning mechanism employed, this probe design can more fully use the effective optical channel area of the probe than any other reported probe design. For example, in our prototype PARS-OCT probe design, the ratio of arc length at the working distance to the probe diameter is 0.56 . We note that our prototype is not optimally designed to achieve the best ratio. In an optimal design with the same scan angle and beam width characteristics, this ratio can reach 3.57 if the GRIN lenses used are swapped for a pair with diameter $112 \mu \mathrm{m}$ and a 31gauge needle is used for the outer needle shaft. Finally, this probe design allows us to obtain a complete volumetric scan of the forward region, because the exit beam direction can be changed freely by rotating the two needles independently.

Based on this probe design, we fabricated a prototype PARS-OCT needle probe and provided a proofof-principle demonstration of the PARS-OCT capability. The prototype consists of an inner needle (18XTW gauge) and an outer needle (16TW gauge) that are cut from standard hypodermic tubings (Poppers \& Sons, Inc.) and attached to a pair of angled GRIN lenses. The overall probe diameter is $1.65 \mathrm{~mm}$. The exposed needle length is $1 \mathrm{~cm}$ in this prototype and can be easily adjusted to be up to $3 \mathrm{~cm}$ long. Both GRIN lenses ( $1 \mathrm{~mm}$ in diameter) are angle cut and polished at an angle $\psi$ of $22^{\circ}$ at a suitable length. The measured working distance is $1.4 \mathrm{~mm}$ (from the exit face of the probe, when the exit beam is straight), and the focused exit beam has a measured focal spot size of $\sim 10.3 \mu \mathrm{m}$. When the exit beam is tilted, the working distance will be slightly shorter $(\sim 1.27 \mathrm{~mm})$, and the focal spot size will be slightly larger $(\sim 12.5 \mu \mathrm{m})$. Two dc motors attached to the proximal end of the probe to provide the rotation actuation. Waterproofing the probe for surgical work can be done through numerous approaches. We evaluated the use of dental epoxy as a way to seal the probe, and it proved to be sufficient; immersing a treated probe in water did not change its performance.

The relationship among $\theta, \zeta_{1}$, and $\zeta_{2}$ defies a simple analytical expression. MATLAB simulation of the probe trajectory during B scans [see Fig. 2(a)] shows a consistent up-and-down sweep trajectory with acceptable deviation (the ratio of the maximum angle deviation to the maximum sweep angle is $1.2 \%$ ). An analytical expression of the $\theta$ as a function of $\zeta_{1}, \zeta_{2}$, assuming $\alpha-\psi$ is small, can be derived as

$$
\theta=-N_{0} \sqrt{A} \sin (Z \sqrt{A}) d \tan (\psi)(\alpha-\psi)+N_{0} \cos (Z \sqrt{A}) \theta_{1},
$$

where

$$
\begin{aligned}
\theta_{1}= & {\left[\left(1-\frac{1}{N_{0}}\right)^{2} \sin ^{2} \psi+2\left(\frac{1}{N_{0}}-\frac{1}{N_{0}^{2}}\right)\right.} \\
& \left.\times(\alpha-\psi) \sin \psi \cos \left(\xi_{1}-\xi_{2}\right)+\left(\frac{\alpha-\psi}{N_{0}}\right)^{2}\right]^{1 / 2}
\end{aligned}
$$

and $\alpha=\sin ^{-1}\left(N_{0} \sin \psi\right) . N_{0}$ and $\sqrt{A}$ are the on-axis refractive index and the index gradient constant of the 
GRIN lens, respectively. $Z$ is the length of the second GRIN lens, and $d$ is the diameter of the GRIN lens. We experimentally verify the agreement of the performance of the prototype probe with this simple formula by experimentally measuring $|\theta|$ versus $\left|\zeta_{1}-\zeta_{2}\right|$. The cases for $\zeta_{1}-\zeta_{2}>0$ and $\zeta_{1}-\zeta_{2}<0$, both shown in Fig. 2(b), indicate very good agreement between theory and experiment.

We next employed the PARS-OCT prototype probe to image the gill structure of a euthanized stage 54 Xenopus laevis tadpole. The OCT engine employed in this experiment is based on a swept laser source with center wavelength $1300 \mathrm{~nm}$, bandwidth $70 \mathrm{~nm}$, power $2.5 \mathrm{~mW}$, sweep rate $250 \mathrm{~Hz}$, and a theoretical signal-to-noise ratio (SNR) of $125 \mathrm{~dB}$. A dual balanced scheme is used to suppress excess noise. ${ }^{9}$ The measured SNR of the PARS-OCT probe system is $93 \mathrm{~dB}$, and the illumination power on the sample is $450 \mu \mathrm{W}$. The drop in the SNR is due to the coupling loss within the probe and the beat noise caused by the interference between the reference beam and internal reflections within the probe.

In the demonstration, we rotated the two needles with equal and opposite angular speeds $(\sim 21 \mathrm{rpm})$ and acquired a single B-scan image from the specimen. We then rotated both needles by $45^{\circ}$ increments and acquired the second, third, and fourth B-scan image [see Fig. 3(b)]. Figure 3(a) shows the photograph of the needle and the tadpole when the images were being acquired. The scanned locations are shown in Fig. 3(b). The acquired images are displayed in Fig. 3(c)-3(f). Each image has $350 \mathrm{~A}$ scan lines and is acquired in $1.4 \mathrm{~s}$. We can clearly discern the gill pockets in the images. The scan depth in the image is $2.3 \mathrm{~mm}$, and the largest scan half-angle is $19^{\circ}$.

The PARS-OCT probe design is capable of performing volumetric scans with very few modifications. By simply incrementally shifting the starting orientations of the two GRIN lenses while performing B-scans, we can acquire volumetric scans. A simpler implementation will be to introduce a slight offset to

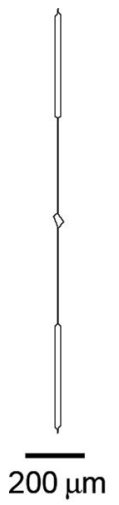

(a)

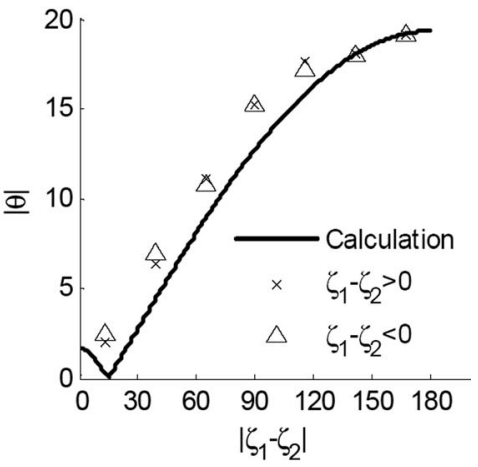

(b)
Fig. 2. (a) Calculated B-scan mode profile as projected in the focal plane of the exit beam. (b) Calculated and measured exit beam polar angle, $|\theta|$, versus the difference between the orientation angles of the two GRIN lenses, $\left|\zeta_{1}-\zeta_{2}\right|$.

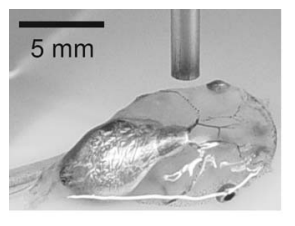

(a)

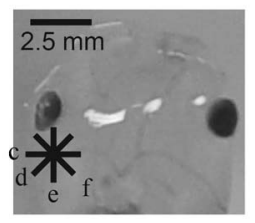

(b)

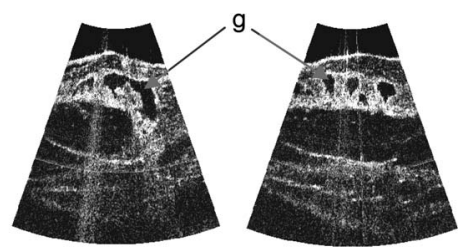

(d)

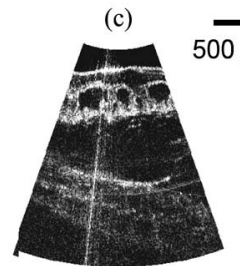

(e)

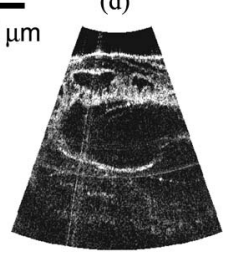

(f)
Fig. 3. OCT images of the gill pockets of a stage 54 Xenopus laevis tadpole. (a) Photograph of the probe and the tadpole when the images are acquired. (b) Indication of scan locations (c)-(f) in the tadpole. (c)-(f) OCT images acquired by the PARS-OCT probe; g, gill pockets.

the relative rotation scan velocities. In this case, the acquired B-scans will automatically sweep through the entire volume scan space. Our acquisition of $\mathrm{B}$-scans in different direction with the prototype probe demonstrates the simplicity by which volumetric scans may be performed.

In summary, we have demonstrated a forwardimaging OCT needle probe with a $1.65 \mathrm{~mm}$ outer diameter based on the use of two rotating GRIN lenses for probe beam scanning. The probe is readily miniaturizable and capable of performing volumetric scans with very few modifications. The probe can be potentially used in needle surgical procedures to provide high-resolution 3D tomographic images of the targets forward of the probe.

This research was supported by funding from the National Institutes of Health, 5R21EB004602-02. J. Wu's e-mail address is jigang@caltech.edu.

\section{References}

1. G. J. Tearney, S. A. Boppart, B. E. Bouma, M. E. Brezinski, N. J. Weissman, J. F. Southern, and J. G. Fujimoto, Opt. Lett. 21, 543 (1996).

2. X. D. Li, C. Chudoba, T. Ko, C. Pitris, and J. G. Fujimoto, Opt. Lett. 25, 1520 (2000).

3. P. R. Herz, Y. Chen, A. D. Aguirre, K. Schneider, P. Hsiung, J. G. Fujimoto, K. Madden, J. Schmitt, J. Goodnow, and C. Petersen, Opt. Lett. 29, 2261 (2004).

4. X. M. Liu, M. J. Cobb, Y. C. Chen, M. B. Kimmey, and X. D. Li, Opt. Lett. 29, 1763 (2004).

5. T. Q. Xie, H. K. Xie, G. K. Fedder, and Y. T. Pan, Appl. Opt. 42, 6422 (2003).

6. T. A. King and G. M. Fuhrman, Semin Surg. Oncol. 20, 205 (2001).

7. W. A. Reed, M. F. Yan, and M. J. Schnitzer, Opt. Lett. 27, 1794 (2002).

8. J. C. Jung and M. J. Schnitzer, Opt. Lett. 28, 902 (2003)

9. M. A. Choma, M. V. Sarunic, C. H. Yang, and J. A. Izatt, Opt. Express 11, 2183 (2003). 